\title{
Die Fabel
}

Liebe Leserinnen, liebe Leser,

der Mensch ist Urheber der Technik. Hat er ein Urheberrecht? Dazu folgende Geschichte: Magnetbänder waren in den 60er/70er-Jahren bevorzugtes Speichermedium. Sie sind spröde im Umgang. Will man etwa einen Datensatz löschen, muss der gesamte Bandspeicher auf ein neues Band kopiert und dabei der betroffene Datensatz ausgelassen werden. Das alte, das Vater-Band, wird zur Sicherheit aufbewahrt. Auf ihm bleibt der zu löschende Datensatz stehen. Erst nachdem es bei der nächsten Änderung zum Großvater geworden ist, wird es für eine anderweitige Verwendung frei gegeben und kann überschrieben, d.h. gelöscht, werden. Das gesetzlich definierte Löschen kann damit um Stunden, aber auch um Jahre, verzögert sein. Das ist nicht zu ändern. Es verstieß aber seinerzeit gegen den Wortlaut des Gesetzes. Als der schriftführende Referent darauf aufmerksam gemacht wurde, reagierte er heftig: „Da müsst ihr euch etwas anderes einfallen lassen. Das Recht gibt vor; die Technik hat zu folgen." Urheberrecht.

Diese Technik hatte gerade die Stecktafeln der Lochkarten- und Tabelliermaschinen durch Computer ersetzt; einer neuen Technik, die auf die Labors des 2. Weltkriegs zurückging und sich in der Wirtschaft etabliert hatte. Die Hersteller warben mit dem Effektivitätszuwachs und polarisierten damit die öffentliche Meinung. Neben den Computerbegeisterten gab es Besorgte. Sie erkannten sich und das Recht als von der Technik gehetzt, wie - häufig zitiert - der Hase in der Fabel, der mit dem Igel andauernd um die Wette läuft, aber immer erst als Zweiter ans Ziel kommt: Zuerst die Technik, dann das Recht. Das war inakzeptabel und beschämend; man war bemüht, das Verhältnis umzudrehen.

Die andere Geschichte: Die Technik war nicht zuletzt mit der Atombombe in die Kritik geraten. Man hatte aber auch ihre beeindruckenden Erfolge gesehen, wie z.B. die Mondlandung. Und hatte gesehen, was zielgerichtete Planung zuwege bringen kann. Als die Glasfasern produktionsreif waren und damit im Postwesen ein neuer Schub anstand, beschloss die Bundesregierung, diesen Schub zu planen. Dazu setzte sie die „Kommission für den Ausbau des technischen Kommunikationssystems (KtK)“ ein. Die arbeitete das Thema wissenschaftlich auf, machte Vorschläge für neue Postdienste und empfahl dazu mehrere Pilotprojekte (siehe auch Kolumne 06/13). Mit innen sollten in einigen ausgesuchten Städten Dienste, wie etwa Bildschirmtext, anwendungsreif entwickelt werden. Danach sollten sie etappenweise als reguläre Dienste auf das Bundesgebiet ausgedehnt werden; man wollte geplanten Fortschritt. Die Bevölkerung sollte das Angebot annehmen; die Dienste sollten in die Gesellschaft organisch einwachsen. Die Projekte wurden begonnen; zum Teil zu Ende geführt. Aber sie wuchsen nicht ein.

Diese Geschichten hängen beide an der Vorstellung: Der Mensch ist Urheber der Technik; es liegt an ihm, sein Urheberrecht auszuüben und über die Technik zu bestimmen. In diesem Geiste war der Datenschutz auf den Weg gebracht worden und die Pilotprojekte planmäßig angelaufen. Aber dann kam das Internet dazwischen; es faszinierte den Markt mit neuen Möglichkeiten und Risiken und lenkte von den hausbackenen neuen Diensten ab. Sie waren schon vor ihrer Einführung obsolet. Das Internet hatte sich dagegen als Wildwuchs bedarfsgetrieben entwickelt und in den Kommunikationsalltag integriert. Es war bald unentbehrlich und schnell allgegenwärtig.

In diesem fabelgleichen Wettlauf war das Recht stets Zweiter, hinter der Technik; sie griff Perspektiven auf, entwickelte Angebote; es gab Probleme; dann erst war das Recht gefragt. Es hätte im Prinzip schon auf die Perspektiven reagieren können, wie die Bundesregierung auf die Erfindung der Glasfasertechnik hin. Doch reagierte - wie immer - der Markt schneller und zielgenauer, selektiv, ungeplant und ethisch unbelastet. Ihn interessiert zuerst das Angebot; es wird von der Technik geliefert. Also erst kommt die Technik. Ich meine, liebe Leserinnen und Leser: das ist einmal so, und ist auch weiter nicht beschämend, solange das Recht auch kommt und angemessen reagiert.

Mit freundlichen Grüßen, Ihr

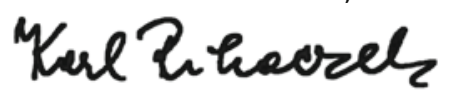

